Jean CÉSAR ${ }^{1,2}$

Jérémy BOUYER ${ }^{1,3}$

Laurent GRANJON ${ }^{4}$

Massouroudini AKOUDJIN ${ }^{1,5}$

Laure GUERRINI ${ }^{1,6}$

Dominique LouPPE ${ }^{7}$

${ }^{1}$ Centre international de recherchedéveloppement sur l'élevage

en zone subhumide

BP 454, Bobo-Dioulasso

Burkina Faso

${ }^{2}$ Cirad

Urp Pôle pastoral en zone sèche Isra-Lnerv, BP 2057, Dakar Hann Sénégal

${ }^{3}$ Cirad

UMR Contrôle des maladies animales exotiques et émergentes

Campus international de Baillarguet F34398 Montpellier Cedex 05

France

${ }^{4}$ Ird

Umr 022 Cbgp

Campus de Bel-Air

BP 1386, Dakar, CP 18524

Sénégal

${ }^{5}$ Institut de développement rural Université polytechnique

de Bobo-Dioulasso,

BP 1091, Bobo-Dioulasso

Burkina Faso

${ }^{6}$ Cirad

Umr Agirs

Campus international de Baillarguet F34398 Montpellier Cedex 05

France

7 Cirad

Ur B\&sef

Campus international de Baillarguet F34398 Montpellier Cedex 05 France

\title{
Les relictes forestières de la falaise de Banfora : un peuplement original au voisinage de Bobo-Dioulasso, Burkina Faso
}

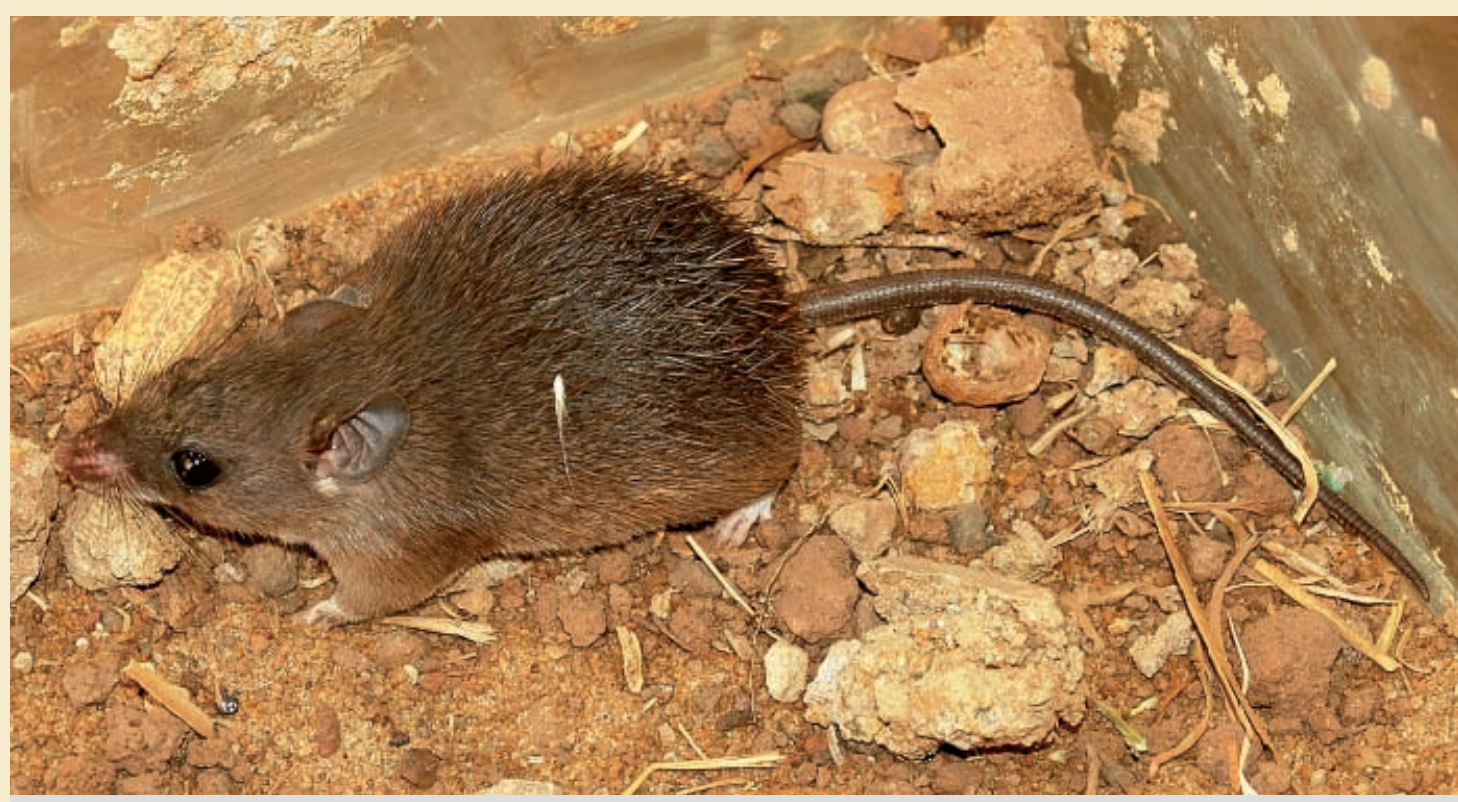

Photo 1.

Acomys johannis.

Photo L. Granjon. 
RÉSUMÉ

\section{LES RELICTES FORESTIÈRES DE LA FALAISE DE BANFORA : UN PEUPLEMENT ORIGINAL AU VOISINAGE DE BOBO- DIOULASSO, BURKINA FASO}

La falaise de Banfora est d'une grande richesse floristique et faunique. Ses forêts renferment des espèces endémiques mais aussi des espèces remarquables telles que Albizia dinklagei, Acridocarpus chevalieri ou Warneckea fascicularis qui ne se retrouvent nulle part ailleurs au Burkina Faso. C'est aussi le cas de certaines espèces d'insectes telles que Dicronorhina kouensis ou Stephanorhina guttata. Toutefois, ces forêts sont menacées, particulièrement autour de Bobo-Dioulasso où la faune d'origine a déjà partiellement disparu. Les sites de la falaise sont comparés entre eux ainsi qu'avec d'autres forêts de la région de Bobo-Dioulasso. Une très grande diversité biologique s'observe d'une forêt à l'autre. La dispersion des espèces suggère que ces forêts résulteraient de masses forestières plus vastes dont elles seraient les relictes. La végétation forestière des falaises aurait trois origines : la forêt dense sèche, caractérisée par Guibourtia copallifera, qui pourrait représenter le fond forestier originel avant l'invasion de la flore des savanes ; la forêt dense humide, représentée par la majorité des espèces ripicoles, qui serait issue de la masse forestière humide en suivant le réseau hydrographique ; un fond de flore montagnarde inféodé aux falaises gréseuses, dont l'unique représentant serait Warneckea fascicularis, auquel il serait possible de rattacher les espèces de rochers. La communauté de rongeurs des sites forestiers de la falaise de Banfora est un mélange d'espèces typiquement forestières (en particulier Praomys rostratus) dans les zones les moins perturbées et d'espèces très anthropophiles (comme Rattus rattus) sur les sites les plus dégradés par l'action humaine. La plupart des espèces d'insectes ont probablement pour origine la forêt humide de Côte d'Ivoire, avec des influences maliennes, comme Coeliades aeschylus, ou togolaises, comme Dicronorhina kouensis, espèces qui se rencontrent surtout en zone de savane guinéenne, à proximité des forêts galeries. Ces espèces n'étant pas observées au Sud, ni respectivement à l'Est et à l'Ouest du Burkina Faso, la falaise de Banfora pourrait constituer un point de rencontre entre différentes faunes, ce qui en fait toute la richesse.

Mots-clés : falaise gréseuse, dynamique, végétation, bioindicateur, micromammifères, insectes.

\section{ABSTRACT}

\section{RELICT FOREST IN THE BANFORA CLIFF: A UNIQUE FOREST COMMUNITY IN THE NEIGHBOURHOOD OF BOBO- DIOULASSO, BURKINA FASO}

The Banfora cliff are home to a wealth of flora and fauna. Endemic species are found in its forests, as well as species of outstanding interest such as Albizia dinklagei, Acridocarpus chevalieri or Warneckea fascicularis, which are found nowhere else in Burkina-Faso. This is also the case with some insect species, such as Dicronorhina kouensis or Stephanorhina guttata. However, these forests are under threat, especially around Bobo-Dioulasso where the native fauna has already become partly extinct. Comparisons were made between the Banfora cliff sites and between these and other forest sites in the Bobo-Dioulasso region. Biological diversity is very high among these forests, and the dispersion of species suggests that they are remnants of much larger forest areas. The forest vegetation on the cliffs appears to be of three types: dense dry forest, characterised by Guibourtia copallifera, which may represent the original old-growth forest stock before it was invaded by savannah vegetation; dense humid forest, represented by most of the riparian species, which probably evolved along the river network from the humid forest mass; and relict old-growth mountain flora that depends on the sandstone cliff environment, with Warneckea fascicularis, to which rock species could be attached, as the sole representative. The rodent community in the Banfora cliff forest sites is a mixture of typically forestdwelling species (particularly Praomys rostratus) in the least disturbed zones and highly anthropophilous species (like Rattus rattus) in the sites most severely degraded by human activities. Most insect species probably originated in the humid forests of Côte d'Ivoire, with some influence from Mali, like Coeliades aeschylus, or Togo, like Dicronorhina kouensis, both of which tend to be found in the Guinean savannah zone close to gallery forests. As these species are neither found in the South, nor respectively in the East and the West of Burkina Faso, the Banfora cliffs may be a meeting point for different types of fauna, hence its outstanding biodiversity interest.

Keywords: sandstone cliff, dynamics, vegetation, bioindicator, small mammals, insects.
RESUMEN

\section{LOS BOSQUES RELICTOS DEL ACANTILADO DE BANFORA, UNA MASA FORESTAL ÚNICA EN LAS CERCANÍAS DE BOBO-DIOULASSO, BURKINA FASO}

El acantilado de Banfora posee una gran riqueza de fauna y flora. Sus bosques encierran especies endémicas, pero también especies notables tales como Albizia dinklagei, Acridocarpus chevalieri o Warneckea fascicularis que no se encuentran en ningún otro sitio más de BurkinaFaso. Lo mismo sucede con algunas especies de insectos tales como Dicronorhina kouensis o Stephanorhina guttata. Sin embargo, estos bosques están amenazados, especialmente alrededor de Bobo-Dioulasso, en donde la fauna original ya ha desaparecido parcialmente. Se comparan los distintos sitios del acantilado entre sí y con algunos bosques más de BoboDioulasso. Se observa una gran diversidad biológica de un bosque a otro. La dispersión de especies deja pensar que estos bosques serían formaciones relictas que provendrían de masas forestales mucho más vastas. La vegetación forestal de los acantilados tendría tres orígenes: el bosque denso seco, caracterizado por Guibourtia copallifera, que podría representar el sustrato forestal antes de la invasión de la flora de sabana; el bosque denso húmedo, representado por la mayoría de especies ripícolas y que procedería de la masa forestal húmeda que fue progresando a través de la red hidrográfica; por fin, un sustrato de flora de montaña, afincado en los acantilados de arenisca y cuyo único representante sería Warneckea fascicularis, al que se le podrían incorporar las especies rocosas de peñascos. La comunidad de roedores de los sitios forestales del acantilado de Banfora es una mezcla de especies típicamente forestales (particularmente Praomys rostratus) en las áreas menos perturbadas y de especies muy antropófilas (como Rattus rattus) en los sitios más degradados por la actividad antrópica. La mayoría de las especies de insectos probablemente sean originarias del bosque húmedo de Côte d'Ivoire, aunque también haya influencias malienses, como Coeliades aeschylus, o togolesas, como Dicronorhina kouensis, especies que se encuentran más bien en zonas de sabana guineana, cerca de los bosques de galería. Al no encontrarse estas especies ni en el Sur, ni respectivamente al Este y al Oeste de Burkina Faso, el acantilado de Banfora podría constituir un punto de encuentro entre distintas faunas, y en esto consiste toda su riqueza.

Palabras clave: acantilado de arenisca, dinámica, vegetación, bioindicador, micromamíferos, insectos 


\section{Introduction}

La falaise de Banfora, qui s'étend de cette ville à Bobo-Dioulasso, est unique au Burkina Faso. Elle abrite un grand nombre de sources d'eau vitales pour la population. Les relictes forestières y sont fréquentes dans des dépressions et des petits canyons dans le bas de la falaise. Beaucoup d'entre elles sont devenues des sites sacrés et des lieux de culte ou de pèlerinage. Enfin, une grande partie des jardins potagers qui nourrissent les villes de BoboDioulasso et de Banfora est alimentée par les cours d'eau issus de la falaise.

Quelques rares études de la diversité biologique ont été réalisées dans cette région pour la végétation (GUINKO, 1985, 2005 ; KIÉMA 2007, KIÉMA, FouRNIER, 2007) et pour la faune (GAUTUN et al., 1985, pour les rongeurs; GARDINER et al., 2005, pour les papillons) ; mais elles n'ont pas été formellement replacées dans un contexte régional. L'article met en perspective les résultats obtenus à partir d'inventaires réalisés dans différents sites de la falaise de Banfora et autour d'elle. Ces résultats montrent que cette région est un carrefour de biodiversité, confirmant la nécessité de la préservation d'un patrimoine naturel et culturel unique pour le pays.

\section{Richesse biologique de la falaise de Banfora}

\section{La flore}

La richesse floristique de cette falaise est exceptionnelle. Dans les galeries, se retrouvent de nombreuses espèces des forêts denses de Côte d'Ivoire telles que Albizia ferruginea (Guill. et Perr.) Benth. (Mimosaceae), Sterculia tragacantha Lindl. (Sterculiaceae), Pentadesma butyracea Sabine (Clusiaceae), et, sur le site sacré de Dafra, le remarquable Albizia dinklagei (Harms) Harms (Mimosaceae).

Les fougères Osmunda regalis L. (Osmundaceae), Bolbitis heudelotii (Bory ex-Fee) Alston (Lomariopsidaceae), se sont réfugiées dans les sources et les cascades. Une espèce endémique, Pandanus brevifrugalis Huynh (Pandanaceae), n'existe qu'à Banfora et à Moussodougou.

Sur les crêtes rocheuses nichent des espèces particulières comme Batopedina tenuis (A. Chev. ex-Hutch. \& Dalz.) Verdc. (Rubiaceae), Monechma ndellense (Lindau) Miège \& Heine (Acanthaceae) ou le rare Acridocarpus chevalieri Sprague (Malpighiaceae). Plus extraordinaire encore, Warneckea fasciculare (Planch. ex-Benth.) Jac.-Fél. (Melastomataceae) n'est à ce jour connu que dans les montagnes d'Afrique de l'Ouest. Il est une véritable relicte d'une flore montagnarde passée ; il faut aller jusqu'au massif de Man en Côte d'Ivoire ou sur le Fouta-Djalon en Guinée pour le retrouver.

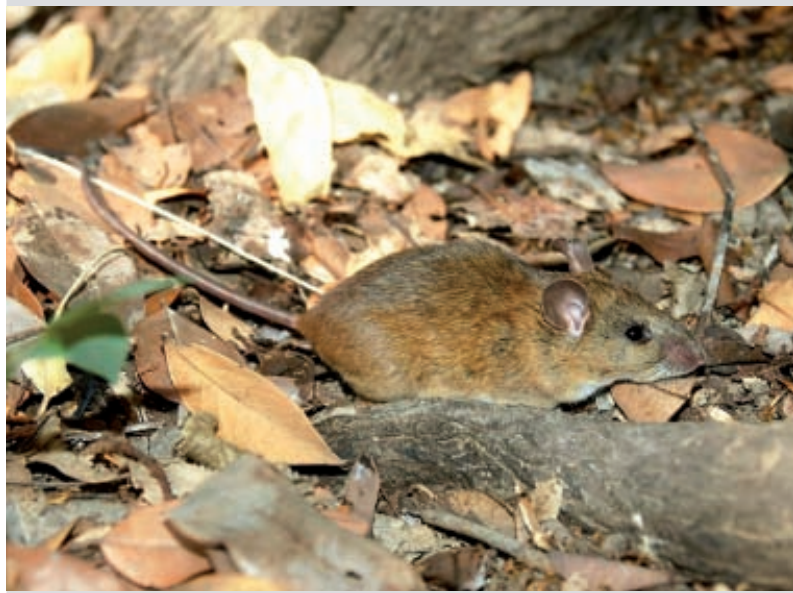

Photo 2.

Praomys rostratus.

Photo L. Granjon.

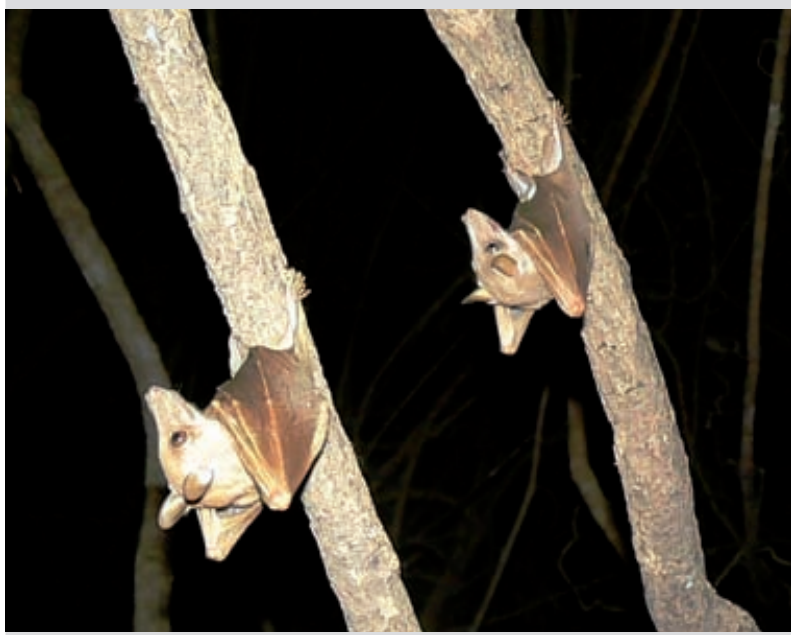

Photo 3.

Epomophorus gambianus.

Photo N. Weber.

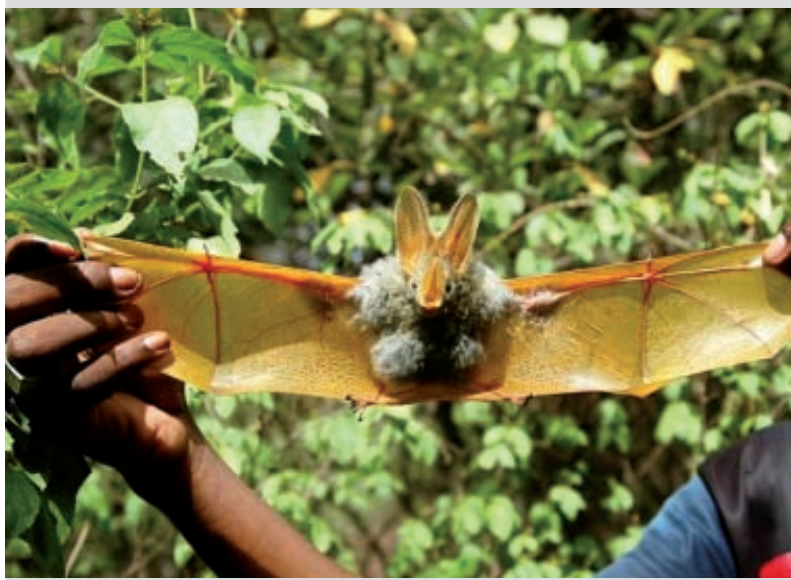

Photo 4.

Lavia frons.

Photo L. Granjon. 

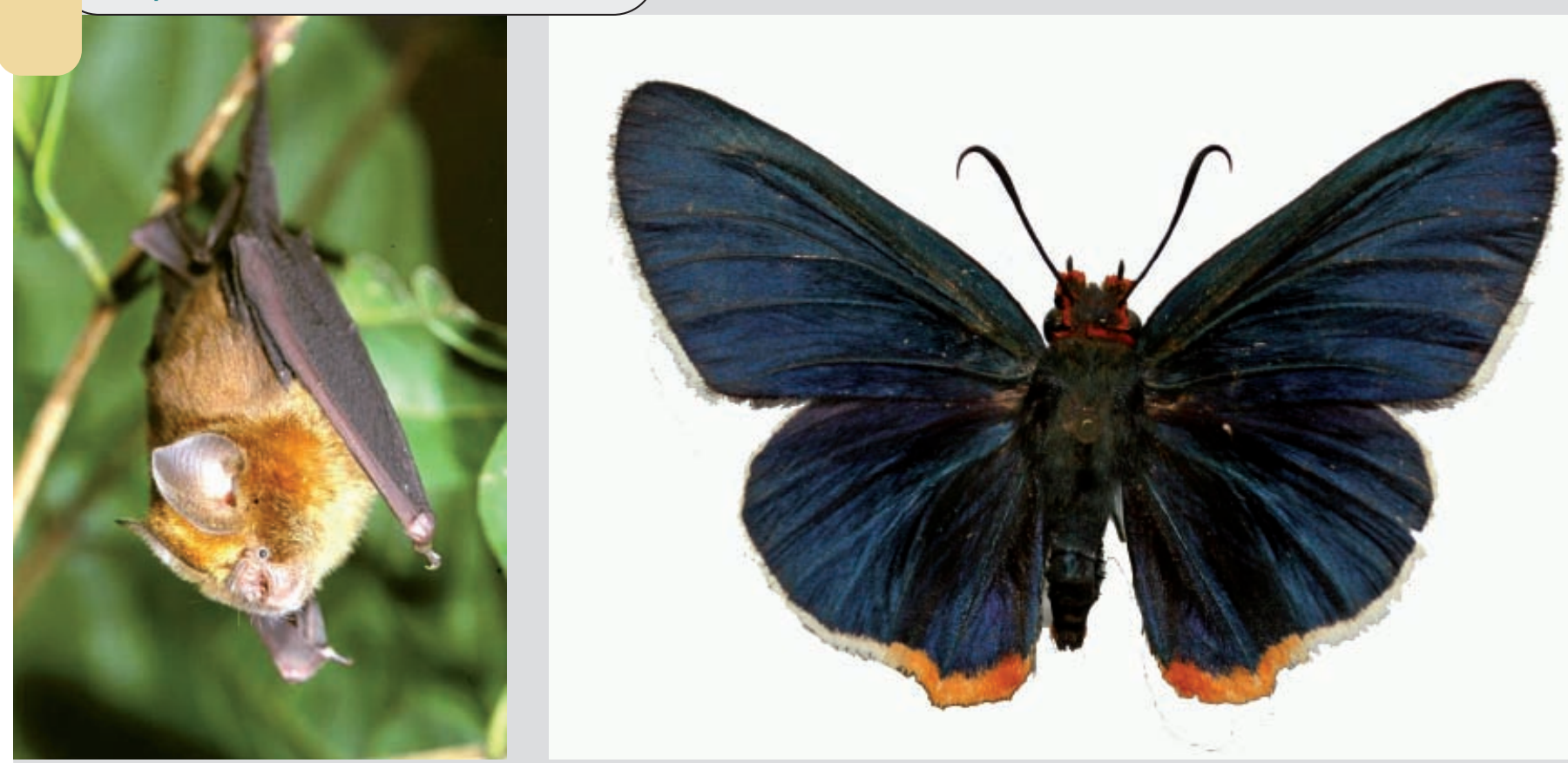

Photo 5 (ci-dessus). Hipposideros ruber. Photo J. Fahr.

Photos 6 (ci-contre). Larve de dernier stade de Coeliades aeschylus sur Acridocarpus chevalieri, site de Dafra, $1^{\text {er }}$ juin 2007, et adulte (ci-dessus) Photos J. Bouyer.

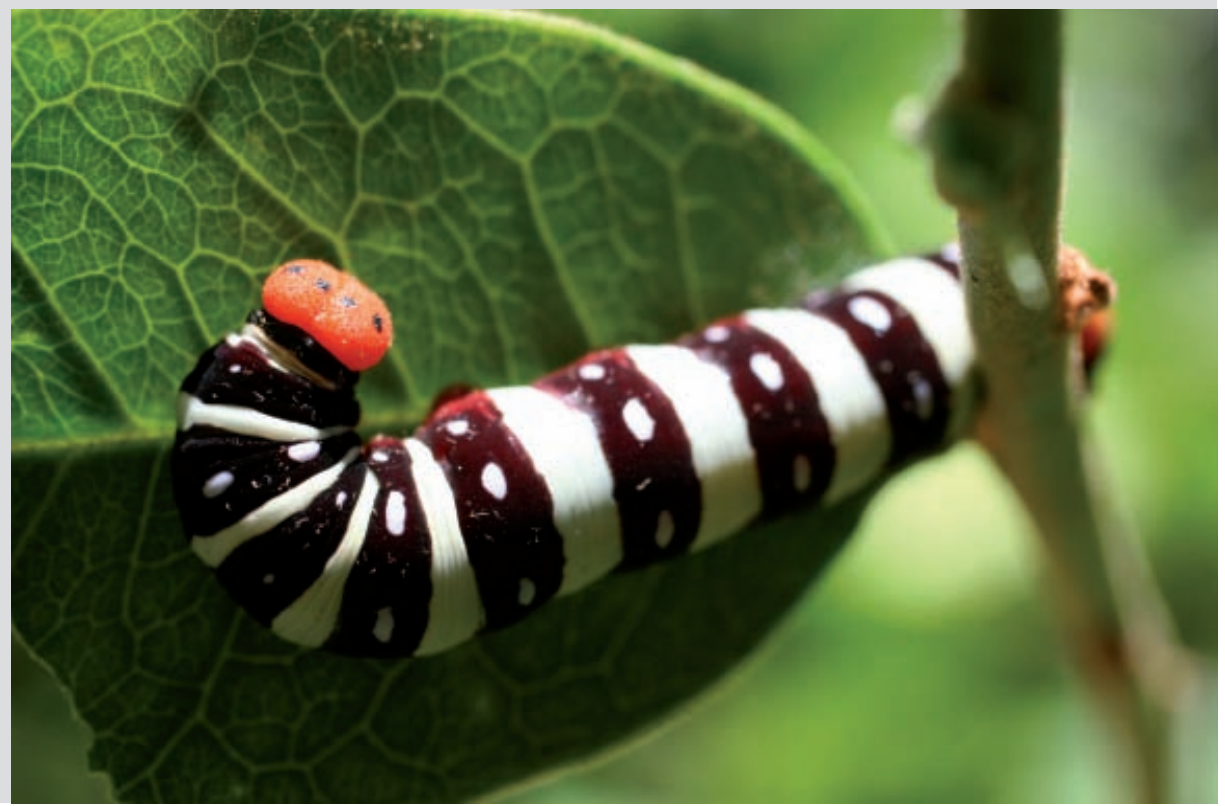

La faune

\section{Les mammifères}

La faune de grands mammifères est aujourd'hui appauvrie et les espèces présentes très discrètes, comme partout ailleurs en Afrique de l'Ouest en dehors des aires protégées. Les grands carnivores ont laissé la place aux petites espèces (mangoustes, genettes, renards), alors que les antilopes ne sont plus présentes qu'en petits effectifs comme, par exemple, le guib harnaché (Tragelaphus scriptus Pallas). Les éléphants (Loxodonta africana Blumenbach) circulent encore dans la plaine en contrebas de la falaise, alors que, parmi les primates, les babouins (Papio anubis Lesson) sont certainement parmi les hôtes les plus réguliers de la falaise. Chez les rongeurs, le rat épineux (Acomys johannis Thomas ; photo 1) est l'espèce la plus étroitement associée aux milieux rocheux ; Praomys rostratus Miller (photo 2) est ici en limite nord de sa répartition en Afrique de l'Ouest et se rencontre seulement dans les fragments de forêt humide peu perturbés par les activités humaines comme à Toussiana, au nord de Banfora. Un cortège d'espèces de chauve-souris est également présent, mélange d'espèces frugivores très communes comme Epomophorus gambianus Ogilvy (photo 3 ) et d'espèces insectivores soit associées à la savane sahélo-soudanienne (Lavia frons Geoffroy ; photo 4), soit plus inféodées à l'habitat forestier (Hipposideros ruber Noack ; photo 5) (Granjon et al., 2009). 


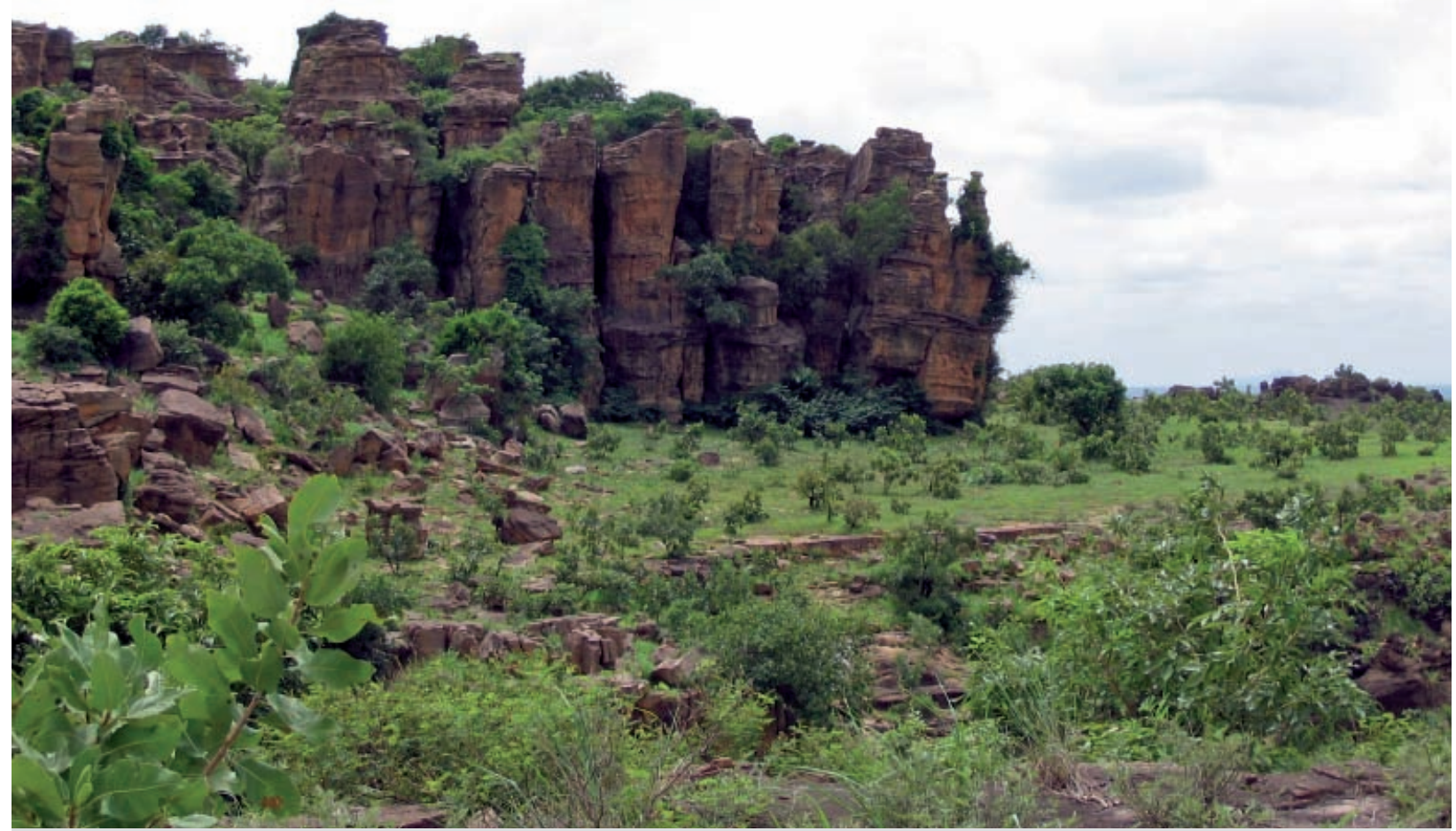

Photo 8.

La falaise près de Dafra ; des bosquets se développent dans les crevasses rocheuses, donnant parfois naissance à des galeries forestières.

Photo J. César.

\section{Principaux sites de la falaise}

La falaise abrite une mosaïque de micro-milieux fragiles et dépendants les uns des autres qu'il est dangereux de perturber ou d'isoler. La destruction des savanes et des bosquets relais accentue la fragmentation des forêts, perturbant la vie de ces écosystèmes tout autant que la destruction des galeries forestières. Cela a été démontré chez Glossina palpalis gambiensis où la fragmentation est associée à une forte différenciation génétique des populations sur de courtes distances géographiques (BOUYER et al., 2009).

L'interprétation des images satellitaires montre que cette continuité forestière est d'ores et déjà menacée au voisinage de la ville de Bobo-Dioulasso (figure 2).

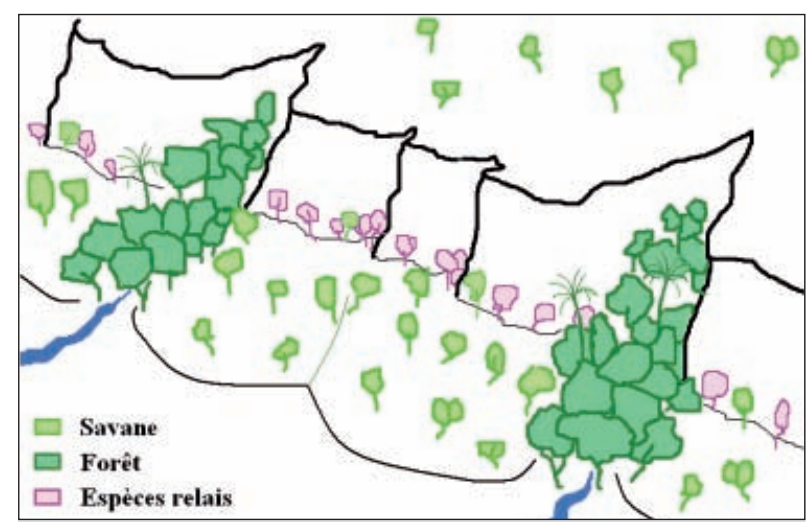

Figure 1.

Discontinuité forestière et bosquets « relais » le long de la falaise de Banfora.

\section{La région de Bobo-Dioulasso}

\section{Le site de Dafra près de Bobo-Dioulasso}

Distant de $6 \mathrm{~km}$ de la ville de Bobo-Dioulasso, le site de Dafra abrite certainement le plus impressionnant de tous les cours d'eau qui traversent la falaise. Partant à mi-hauteur au fond d'une gorge encaissée, le cours d'eau resurgit, formant un micro-lac permanent où logent les silures sacrés (Clarias sp.), s'échappe en un court torrent (fréquenté par des poissons du genre Aplochelichtys), puis s'élargit en un cours d'eau calme et permanent qui irrigue des vergers et des parcelles de maraîchage. Le caractère encaissé et inquiétant laisse percevoir une explication à la sacralisation du site. Celui-ci est très fréquenté par les habitants de Bobo-Dioulasso qui viennent par exemple faire des offrandes aux silures sacrés pour favoriser un enfantement. Cette fréquentation et les déchets alimentaires des offrandes ont probablement favorisé la présence du rat noir (Rattus rattus Linnaeus), espèce commensale par excellence.

Le microclimat et la permanence de l'eau dans l'étroite gorge de Dafra favorisent la végétation forestière qui est dominée par Berlinia grandiflora (Vahl) Hutch. \& Dalz. (Caesalpiniaceae), accompagné de Synsepalum pobeguinianum, (Pierre ex-Lecomte) Aké Assi \& L. Gaut. Manilkara multinervis, Ficus lutea Vahl. (Moraceae), avec un sous-bois de Macrosphyra longistyla (DC.) Hiern (Rubiaceae), Psydrax horizontalis (Schum. \& Thonn.) Bridson (Rubiaceae) et des lianes 
Tetracera alnifolia Willd. (Dilleniaceae), Landolphia heudelotii DC. (Apocynaceae), Apodostigma pallens (Planch. ex-Oliv.) Wilczek (Hippocrateaceae), etc. La fougère Bolbitis heudelotii pousse dans la zone torrentielle. Phyllanthus muellerianus (O. Ktze.) Exell (Euphorbiaceae) et quelques espèces indicatrices de végétation dégradée commencent à apparaître

Trois espèces végétales remarquables croissent sur ce site : dans la partie la plus large de la forêt, Albizia dinklagei, arbre des forêts denses humides; sur les flancs de la gorge encaissée qui conduit au lac, Acridocarpus chevalieri et Warneckea fascicularis. La seconde est fréquente sur les plateaux gréseux du Mali et se trouve dispersé sur la falaise dans des situations abritées. La troisième est une orophile du FoutaDjalon qui se retrouve sur les montagnes de Sierra Leone ou de l'Ouest de la Côte d'Ivoire. À ces espèces s'ajoute la liane herbacée des forêts denses guinéennes, Triclisia patens Oliv. (Menispermaceae). Une seule espèce forestière d'insecte est trouvée à de très faibles densités dans ce site, Chlorochala africana, la cétoine forestière probablement la plus répandue et résistante de l'Afrique de l'Ouest. C'est en revanche le seul site où le lépidoptère Coeliades aeschylus a été observé sur la falaise, grâce à la présence de sa plante hôte.

\section{Le site de Koro}

Le village de Koro se situe à $10 \mathrm{~km}$ de Bobo-Dioulasso. La galerie forestière la mieux développée est en aval de la cascade de Kou, au pied de laquelle quatre fougères (Bolbitis heudelotii, Nephrolepis undulata, Cyclosorus dentatus et surtout Osmunda regalis) s'accrochent à la roche. La galerie forestière est à dominance de Berlinia grandifolia, avec Manilkara multinervis, Elaeis guineensis, Raphia sudanica, Syzygium guineense et, dans le sous-bois, Garcinia ovalifolia, Psychotria psychotrioides.

Dans les galeries voisines, se rencontrent deux espèces remarquables, Sterculia tragacantha et Gardenia imperialis, absentes des autres galeries. Chez les insectes, encore deux espèces de cétoines forestières sont observables, Chlorocala guerini et Eudicella daphnis, à des densités toutefois très faibles. En revanche, le rat noir ( $R$. rattus) est l'espèce dominante chez les rongeurs, au détriment des espèces locales. Parmi ces dernières, c'est Mastomys erythroleucus Temminck, l'espèce la plus ubiquiste en Afrique sahélo-soudanienne, qui est présente.

Une question se pose : les sites de Dafra et de Koro, proches de Bobo-Dioulasso, sont-ils déjà dégradés en raison de la proximité de la grande ville?

\section{La région de Banfora-Toussiana}

\section{Le site de Toussiana}

La cascade de Toussiana, située à 60 km au sud de BoboDioulasso et assez éloignée de Banfora, appartient au même système de falaises (photo 9). Le site comprend une galerie forestière s'élargissant en forêt-galerie en aval de la cascade (photo 10). Il est bordé de savanes arborées à arbustives sur les versants et les éboulis. Le site naturel est très comparable aux systèmes étudiés autour de Koro et de Dafra. Bien que parcouru par le bétail, il n'est que très partiellement cultivé et apparemment suffisamment préservé pour servir de témoin par rapport à Dafra.

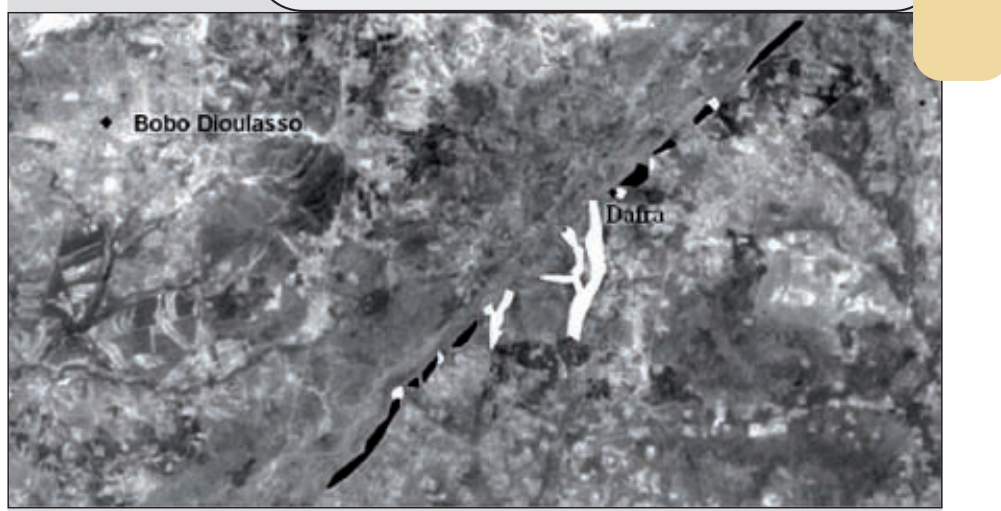

Figure 2.

Analyse d'une image Landsat Etm+ (canaux 4/3/2) de la falaise de Banfora au voisinage de la ville de Bobo-

Dioulasso : en blanc la forêt et en noir les zones avec des espèces relais. Sur cette image, les savanes apparaissent en gris foncé et les cultures en gris clair. Elles montrent l'importance de l'emprise agricole.

Sa forêt galerie est dominée par Berlinia grandifolia, Syzygium guineense (Willd.) DC. var. guineense (Myrtaceae) et Uapaca togoensis Pax (Euphorbiaceae) ; s'y trouvent aussi Carapa procera DC. (Meliaceae), Pterocarpus santalinoides L'Hér. ex-DC. (Fabaceae) et Elaeis guineensis Jacq. (Arecaceae). Dans la strate arbustive, Garcinia ovalifolia Oliv. (Clusiaceae) et Ixora brachypoda DC. (Rubiaceae) abondent. Voacanga thouarsii Roem. \& Schult. (Apocynaceae), Premna lucens A. Chev. (Verbenaceae), la fougère Adiantum schweinfurthii Kuhn (Adiantaceae) accrochée aux escarpements et, en savane, Isoberlinia tomentosa (Harms) Craib \& Stapf (Caesalpiniaceae), Aframomum alboviolaceum (Ridley) K. Schum. (Zingiberaceae) et Solenostemon chevalieri Briq. (Lamiaceae) sont des espèces qui n’ont pas été rencontrées à Koro et Dafra.

Le peuplement de rongeurs de ce site n'est pas particulièrement riche, mais comprend l'espèce forestière Praomys rostratus, ainsi que l'écureuil arboricole Heliosciurus gambianus Ogilby, en plus de l'espèce terrestre Xerus erythropus E. Geoffroy fréquente sur les flancs de la galerie forestière. Chez les insectes, sont également observables les cétoines Chlorochala africana Drury en densité élevée, et Stephanorhina guttata, propre à ce site, ainsi que trois espèces de charaxes forestiers (Charaxes tiridates tiridates Cramer, C. numenes numenes Hewitson et $C$. brutus brutus Cramer).

\section{Le site de Dramandougou}

Très proche de Toussiana, cette galerie est nettement moins développée mais renferme cinq espèces ligneuses qui n'ont pas encore été rencontrées sur la falaise : Pentadesma butyraceae, Albizia ferruginea, Milicia excelsa (Welw.) Berg (Moraceae), Leptoderris brachyptera (Benth.) Dunn. (Fabaceae) et Voacanga africana Stapf (Apocynaceae).

\section{Le site de Banfora}

Le site de Banfora est un marécage au pied de la falaise, caractérisé par la présence d'un poisson endémique, Roloffia banforensis (VoN LOTHAR, 1982). Deux espèces savanicoles, l'orchidée Eulophia juncifolia Summerh. (Orchidaceae) et la graminée Andropogon macrophyllus Stapf (Poaceae), n’ont pas été observées sur le reste de la falaise. 


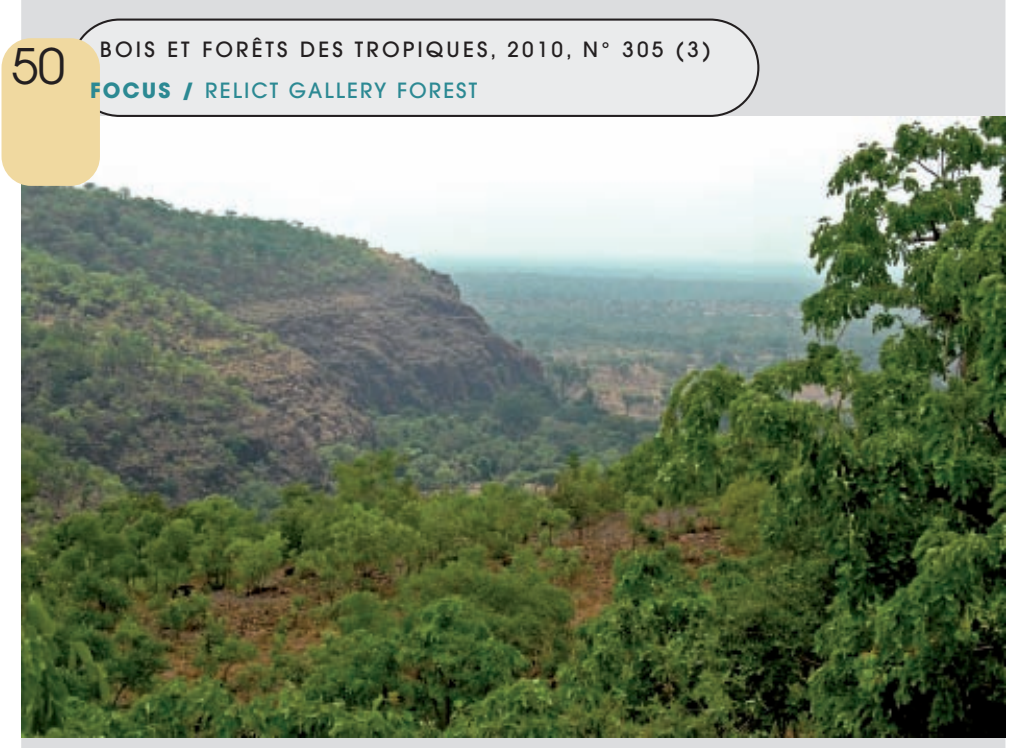

Photo 9.

La falaise près de Toussiana.

Photo L. Granjon.

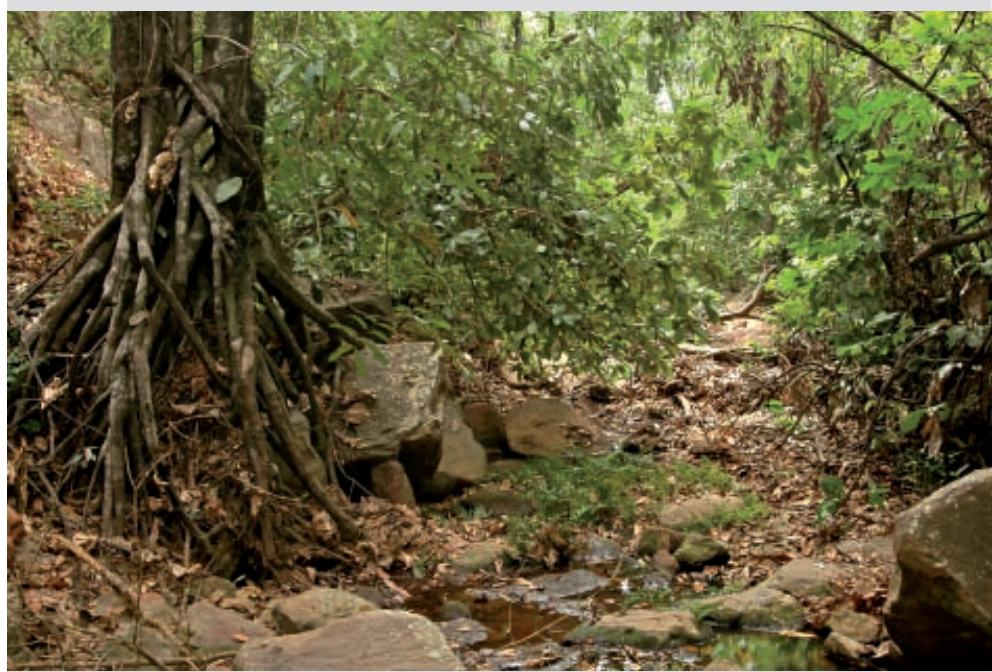

Photo 10.

La galerie forestière de Toussiana en aval de la cascade. Photo L. Granjon.

\section{Le site de Karfiguéla}

La galerie forestière de Karfiguéla, site réputé pour les chutes de la Comoé, est encore plus au sud. À titre de comparaison, sans avoir fait de relevé exhaustif, quelques espèces sont notées qui n'ont été vues ni à Koro ni sur le site de Toussiana : Antiaris toxicaria Lesch. (Moraceae), Pandanus brevifrugalis, Uapaca heudelotii Baill. (Euphorbiaceae), Croton pseudopulchellus Pax (Euphorbiaceae) et Breonadia salicina (Vahl) Hepper \& Wood (Rubiaceae).

\section{Comparaison des deux zones}

La comparaison des flores des deux zones, celle, naturelle, de Banfora-Toussiana et celle de Dafra-Koro, proche de Bobo-Dioulasso, peut-elle renseigner sur le degré de dégradation de la seconde ? Pour ce qui est de la végétation, les richesses floristiques sont sensiblement les mêmes: 137 espèces à Toussiana, auxquelles s'ajoutent 17 espèces sur le site voisin de Dramandougou ; 156 espèces à DafraKoro dont au moins 6 espèces de dégradation.
La comparaison des espèces n'apporte pas non plus de réponse décisive. En effet, rien ne prouve que les espèces de Toussiana absentes de Koro y aient existé dans un passé proche : la différence de latitude, bien que faible, entre Toussiana (1 $050 \mathrm{~mm}$ de pluviosité annuelle) et Koro (1 $000 \mathrm{~mm}$ ) est suffisante pour expliquer l'absence à Dafra de plantes à la limite de leur aire, telles que Aframomum alboviolaceum ou Premna lucens. Il ne s‘agirait pas ici de dégradation mais de limite climatique. Mais cet argument ne peut s'appliquer à toutes les espèces. Isoberlinia tomentosa, par exemple, qui abonde à Farako-Ba près de BoboDioulasso, est connu à Boromo plus au nord-est (HUTCHINSON, DALZIEL, 1958).

Ainsi, Pandanus brevifrugalis est endémique à Karfiguéla. Croton pseudopulchellus fréquente les falaises gréseuses du Mali au Nigeria (BoudET, LEBRUN, 1986) et a été récolté sur les rochers de Fô au nord de Bobo-Dioulasso (AubrévilLe, 1950) ; son absence à Dafra ne peut être climatique.

Antiaris toxicaria est choisi par Guinko (1999), avec Milicia excelsa, comme caractéristique du groupement des relictes boisées ou bois sacrés des districts de l'Ouest du Mouhoun et de la Comoé. Antiaris toxicaria est sans doute proche de la limite de son aire puisque AubréviLLE (1950) l'indique comme relicte des forêts denses des régions montagneuses et le cite dans les systèmes rocheux des monts Mandara au Cameroun au Nord du 10e parallèle.

Selon Aubréville (1959), Uapaca heudelotii remonte bien plus au nord, entre le $11^{\mathrm{e}}$ et le $12^{\mathrm{e}}$ parallèle ; son habitat ripicole le rend peu sensible aux variations climatiques. Il en est de même de Breonadia salicina qui croît sur le lit rocheux des rivières, au Mali de Sikasso à Doniana (Boudet, LeBrun, 1986), à la cascade de Tourny (AubréVILle, 1950), autour de Banfora, à Kampti au sud de Gaoua (LEBRUn et al., 1991) et que nous avons retrouvé à Dindéresso. Plus à l'est, son aire s'étend sur les monts Atakora, au Bénin-Togo (AubréVILLE, 1950), mais il ne semble pas remonter plus au nord.

Si l'argument climatique ne tient pas, comment expliquer la variabilité entre les sites ?

La présence à Dafra d'espèces qui ne se trouvent pas à Toussiana montre tout l'intérêt de cette relicte et laisse supposer que la flore passée y était plus riche et plus diversifiée. Elle devait aussi bénéficier d'une écologie particulière.

En effet, à Dafra, la section permanente du lit du cours d'eau, alimentée par des eaux souterraines, est plus large qu'à Toussiana. La forêt de Dafra n'est pas plus large mais sans doute plus humide à l'origine que celle de Toussiana. La gorge plus étroite y offre un microclimat plus frais même en saison sèche. Cela expliquerait la présence d'Albizia dinklagei, arbre des forêts denses, et même de Warneckea fascicularis et Acridocarpus chevalieri sur les escarpements bénéficiant d'une atmosphère plus humide. D'où la fragilité de la relicte qui n'est plus maintenant protégée par les savanes arborées environnantes qui ont été détruites.

Sur le plan floristique, aussi, un grand nombre d'espèces rudérales et de dégradation est plus remarquable à Dafra qu'à Toussiana, trahissant une fréquentation plus grande par l'homme, notamment parmi les espèces savanicoles, par suite de la mise en culture généralisée des 
savanes. Mais l'analyse statistique ne permet pas de séparer les différentes forêts qui s'échelonnent le long de la falaise car les relevés qui montrent une dégradation sont tous situés en savane (figure 3).

Il semble que les différentes relictes forestières qui se succèdent le long de la falaise, tout en ayant un fond forestier commun (Berlinia grandiflora, Manilkara multinervis, Garcinia ovalifolia), se distinguent chacune par quelques espèces particulières que l'on ne retrouve pas dans les autres sites.

Ainsi, les trois espèces endémiques de Dafra, Albizia dinklagei, Warneckea fasciculare et Acridocarpus chevalieri, n'ont pas été retrouvées ailleurs. Mais Albizia ferruginea, Pentadesma butyracea et Voacanga thouarsii qui se trouvent à Dramandougou (première galerie au nord de celle de Toussiana) sont absentes de Toussiana, Dafra et Koro. Sterculia tragacantha, abondant à Koro, et Gardenia imperialis K. Schum. (Rubiaceae), présent dans deux galeries de Koro, ne sont ni à Toussiana ni même à Dafra pourtant si proche. Croton pseudopulchellus et Antiaris toxicaria, présents à Karfiguéla, n'ont pas été vus sur le reste de la falaise.

Il semble que la richesse ancestrale ait été bien plus grande, et que, par suite de la réduction et de la fragmentation des forêts, un grand nombre d'espèces aient été éliminées, ne laissant dans chaque site que les mieux adaptées aux conditions microclimatiques ou édaphiques locales : durée de l'écoulement du cours d'eau, largeur des plans d'eau, permanence de l'eau dans le sol, encaissement de la vallée, pente, profondeur du sol, etc.

Pour AuBRÉVILLE (1958), les ruptures d'aire peuvent avoir deux explications : vestige d'aire ancienne plus vaste et continue, ou propagation sur des terres favorables, par les animaux notamment. Les deux ont pu jouer dans le temps ; mais il semble que dans la phase actuelle la première hypothèse soit plus probable que la seconde.

Le peuplement de rongeurs, relativement peu diversifié, présente toutefois des différences locales nettes. Ainsi les zones arborées du site de Toussiana sont-elles dominées par une espèce typiquement forestière ( $P$. rostratus), alors que celles de Koro sont clairement occupées par des espèces soit généraliste ( $M$. erythroleucus), soit nettement anthropophile ( $R$. rattus). Ces différences traduisent sans aucun doute des degrés de perturbation très distincts entre ces sites, liés à leur plus ou moins grande proximité avec la ville de Bobo-Dioulasso.

En ce qui concerne les insectes, alors même que les espèces étudiées sont capables de voler d'un site à l'autre, une situation similaire est observable avec un nombre limité d'espèces forestières par site. La fragmentation des bosquets ligneux relais évoquée plus haut est probablement responsable d'une limitation de la dispersion entre sites, comme c'est le cas des glossines, alors que la petite taille des populations dans les fragments résiduels conduit à l'extinction naturelle d'une partie des espèces (HANSKI, GAGGIOTTI, 2004). Une diminution de la biodiversité d'environ $25 \%$ a ainsi été montrée récemment dans un fragment forestier de 1,2 $\mathrm{km}^{2}$ au Ghana, ce qui est pourtant bien plus grand que les lambeaux forestiers de la falaise de Banfora (LARSEN et al., 2009).

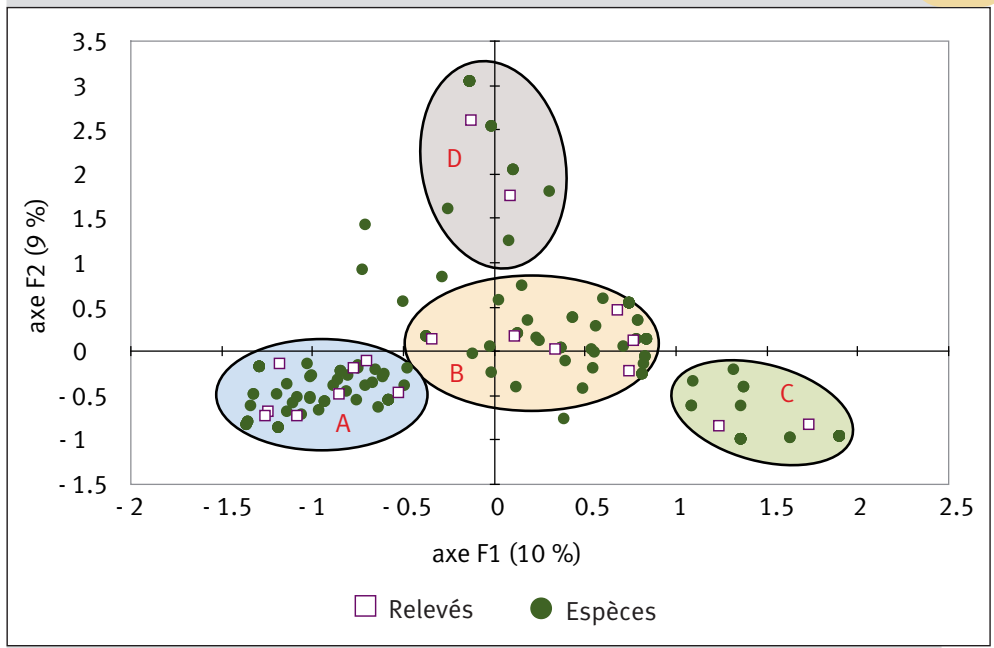

Figure 3.

Typologie de la végétation de Dafra et de Toussiana par l'analyse factorielle des correspondances.

L'axe 1 caractérise la fermeture du milieu et l'axe 2 le niveau d'anthropisation. Quatre groupes apparaissent : les forêts denses (A), les savanes arborées et forêts claires (B), les savanes herbeuses sur lithosol gréseux (C) et les savanes anthropiques (D) caractérisées par des espèces introduites par l'homme.

\section{La dispersion des espèces et l'origine des peuplements}

\author{
La végétation
}

La figure 4 cartographie quelques espèces illustrant la dispersion des espèces de la falaise et aide à expliquer l'origine éventuelle de son peuplement. Les lignes sur la carte représentent des liaisons et non des flèches car le sens de la migration des espèces n'est pas l'objet de l'étude.

Deux espèces des falaises, Acridocarpus chevalieri et Croton pseudopulchellus, sont liées aux massifs gréseux du Mali. Des plantes de l'ancien fond des forêts denses sèches peuvent aussi provenir de ce pays, comme Guibourtia copallifera Benn. (Caesalpiniaceae) (JAEGER, 1956 ; SCHNELL, 1971 ; NASI, SABATIER, 1988), qui est présent à Sindou (LEBRUN et al., 1991), sur les escarpements gréseux de la cascade de Tourni, en peuplement dense dans la forêt de Diéfoula à Folonzo, mais curieusement absent des sites que nous avons visités sur la falaise de Banfora. Si des stations isolées comme Sindou et Tourni peuvent faire songer à une propagation par sauts (seconde hypothèse d'AUBRÉVILLE, 1958), la belle formation quasi monospécifique de Folonzo ne laisse guère de doute sur l'origine climacique de cette rare relicte de forêt dense sèche. Cela est confirmé par l'étude statistique. L'analyse factorielle des correspondances appliquée à six stations parmi les plus représentatives de ces lambeaux forestiers isole de façon très nette la forêt à Guibourtia copallifera de toutes les autres formations étudiées (figure 5). Ces dernières se répartissent suivant l'axe 2 de la figure, le long du gradient latitudinal. 


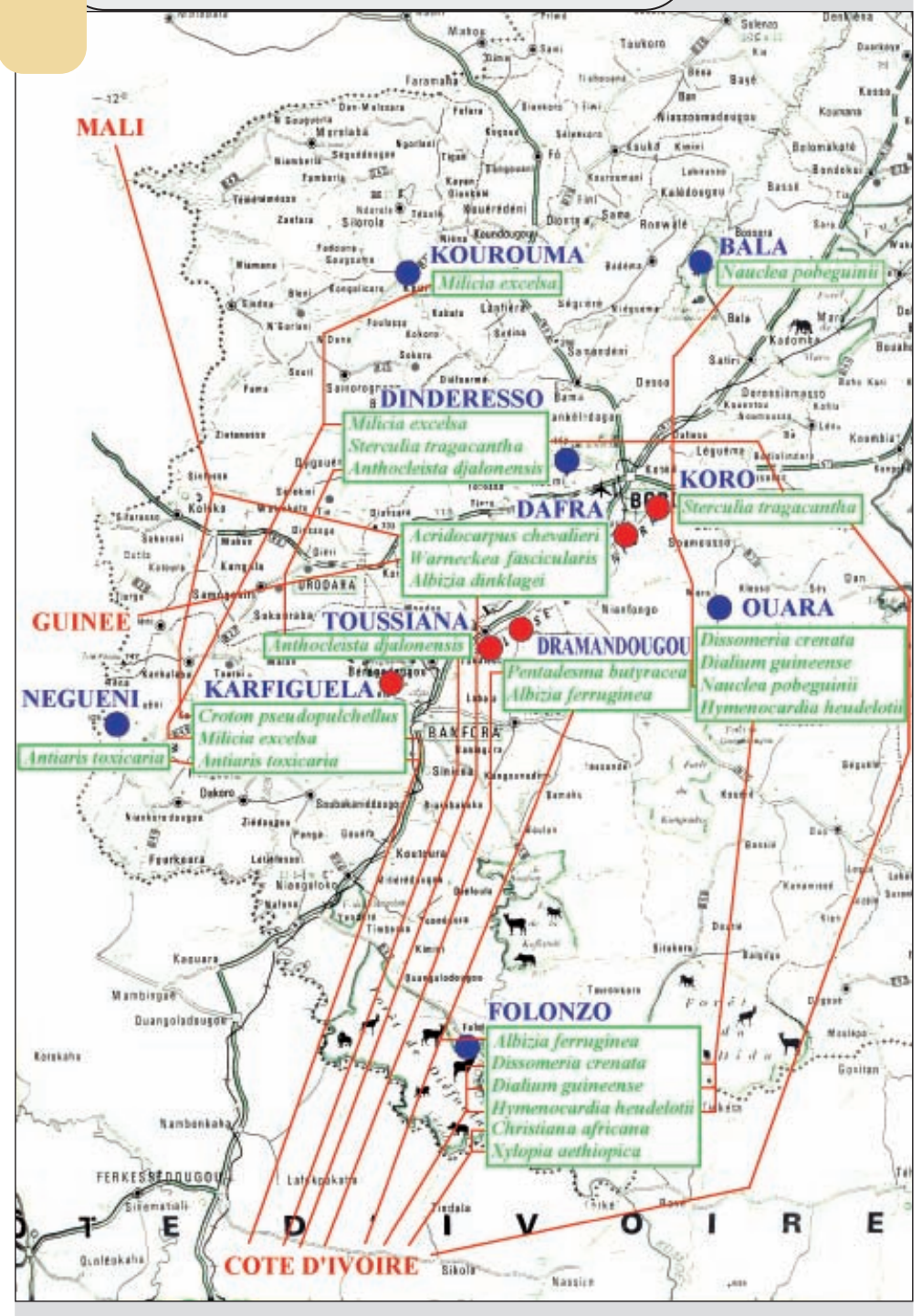

Figure 4.

L'origine supposée du peuplement végétal de la falaise de Banfora. En gris les sites de la falaise, en noir les autres sites.

Warneckea fasciculare se rattache aux montagnes de Guinée ou de l'Ouest de la Côte d'Ivoire. Le site rocheux de Dafra où cette plante a été récoltée se sépare dans l'analyse statistique des autres galeries forestières dans le plan des axes 2 et 3, le troisième axe traduisant la largeur de la galerie (figure 6 ).

De la Côte d'Ivoire ou de l'Est du Ghana proviennent vraisemblablement la majorité des espèces forestières. Alors que certaines espèces comme Christiana africana DC. (Tiliaceae), Xylopia aethiopica (Dun.) A.Rich. (Annonaceae) ou Strychnos cuminodora Leeuwenberg (Loganiaceae) (TEERWENBERG, 1965) ne semblent pas dépasser la région de Folonzo, d'autres comme Milicia excelsa remontent jusqu'à Dindéresso ou même plus haut jusqu'à Kourouma.

Antiaris toxicaria présent à Banfora s'est réfugié dans les falaises de Néguéni près de Loumana, où le site correspond tout à fait à l'écologie extrême décrite par AUBRÉVILLE (1950) comme limite de son aire. II ne semble pas remonter beaucoup plus haut, ce qui reste à vérifier. Les localités citées par BÉGUÉ (1937) ne dépassent pas la latitude de Bobo-Dioulasso.

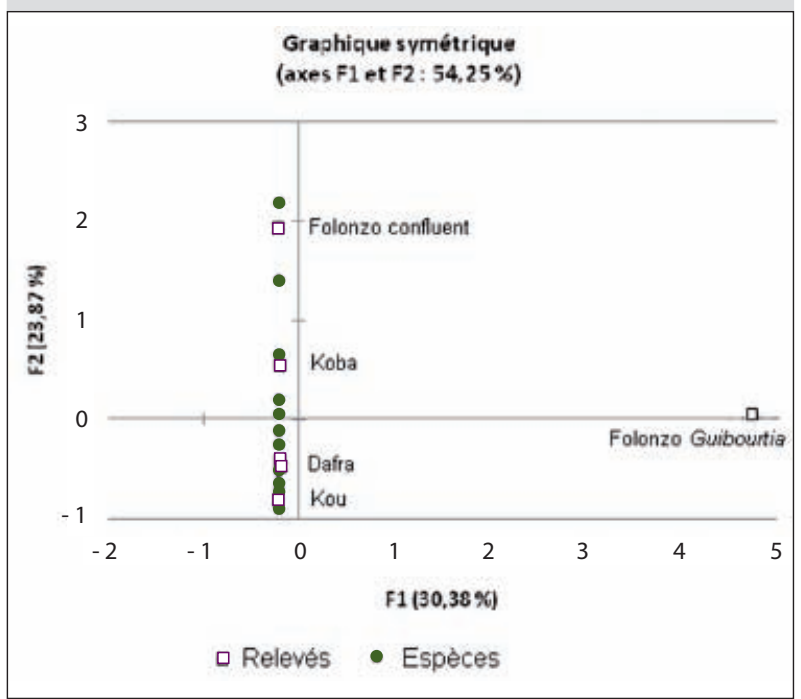

Figure 5.

Typologie de la végétation de six formations de forêt dense, axes 1 et 2 . L'axe 1 isole la forêt dense sèche à Guibourtia copallifera. Les autres stations, qui réunissent des forêts ripicoles, se répartissent le long de l'axe 2 suivant un gradient nord-sud.

La forêt de Folonzo est un relais pour Dialium guineense Willd. (Caesalpiniaceae) et Dissomeria crenata Hook.f. ex-Benth. (Samydaceae), qui atteignent Ouara, mais non la falaise de Banfora, ainsi que pour Albizia ferruginea présent lui près de Toussiana à Dramandougou. De même, des galeries telles que celles de la Koba à Ouara ont pu servir de relais à Cola laurifolia Mast. (Sterculiaceae) ou Diospyros elliotii (Hiern) F. White (Ebenaceae), que nous avons retrouvé bien plus au nord à Koumbia, ou à Nauclea pobeguinii (Pobeguin ex-Pellegr.) Petit (Rubiaceae) présent à Leyessa.

Cependant, ces relais n'expliquent pas tout. Albizia dinklagei et Pentadesma butyracea n'ont été rencontrés dans aucun site visité autre que ceux cités de la falaise, Dafra et Dramandougou respectivement.

Enfin, Sterculia tragacantha, qui se trouve à Dindéresso (GUINKO, 2005) et à Koro, et qui n'a pas été vue dans les autres sites de la falaise - ni à Ouara, ni à Folonzo -, pourrait indiquer des faciès plus humides (NASI, SABATIER, 1988).

La seconde hypothèse d'AUBRÉVILLE paraît plus séduisante pour certaines espèces comme Albizia dinklagei, Albizia ferruginea ou Pentadesma butyracea, dont les fruits peuvent être disséminés par les animaux, y compris les éléphants (AlEXANDRE, 1978). Mais pour Guibourtia copallifera et pour la majorité des espèces dominantes des forêts ripicoles, l'hypothèse de forêts relictes issues d'une masse forestière plus vaste et floristiquement plus riche reste fortement probable. Cela vient renforcer les propositions de GUINKO (1985) et confirmer l'hypothèse d'AUBRÉVILLE (1949) sur l'origine des savanes pour cette zone climatique.

Toutefois, la végétation des systèmes gréseux de Banfora-Bobo-Dioulasso relèverait de trois origines, de la confrontation de trois entités floristiques: 
- La forêt dense sèche, caractérisée par Guibourtia copallifera, qui serait le fond forestier originel avant l'invasion de la flore pyrophile des savanes et forêts claires ;

- La forêt dense humide, représentée par Albizia dinklagei et la majorité des espèces forestières des formations ripicoles, qui serait issue de la masse forestière humide en suivant le réseau hydrographique et contemporain de la remonté vers le nord de ces masses forestières elles-mêmes, donc actuel (ADJANOHOUN, 1964 ; HIERNAUX, 1975 ; Peltre, 1977) ;

- Enfin, un fond de flore montagnarde inféodé aux falaises gréseuses, sans doute plus vulnérable, dont l'unique représentant observé serait Warneckea fasciculare, accroché aux escarpements de Dafra, et auquel pourraient se rattacher les espèces de rochers, Croton pseudopulchelus, Acridocarpus chevalieri, Monechma ndellense et Batopedina tenuis.

À ces trois entités spécifiques des falaises, il faut bien sûr ajouter la flore pyrophite des savanes et des forêts claires, liée à l'homme cette fois, qui ne diffère pas ou peu de la masse des savanes et forêts claires environnantes, qui couvraient naguère la plus grande partie du territoire.

Les espèces relictes de la falaise de Banfora, par leur disposition et leur répartition, tendent à prouver à la fois l'ancienneté de leur origine, l'état de dislocation actuel des masses forestières et leur pérennité en péril.

\section{La faune}

\section{Les rongeurs}

La figure 7 recense les espèces de rongeurs rencontrées dans les galeries forestières échantillonnées dans quatre sites de la falaise de Banfora et autour d'elle. La diversité y apparaît globalement faible (six espèces au total), et composée d'espèces à assez large distribution en Afrique (les deux espèces de Praomys pouvant être considérées comme des "endémiques ouest-africains "), à l'exception de Rattus rattus (le rat noir), espèce aujourd'hui cosmopolite introduite par les Européens à leur arrivée en Afrique de l'Ouest et qui est à tendance invasive en Afrique. Les différences entre sites apparaissent directement liées au degré de perturbation des zones forestières concernées : ainsi, le site de Toussiana, le mieux préservé, est le seul à abriter l'espèce la plus typiquement forestière (Praomys rostratus), dont la population identifiée la plus proche se retrouve dans la forêt classée du Kou à l'ouest de Bobo-Dioulasso (Nicolas et al., 2008), connue pour représenter une relicte de zone guinéenne dans cette région. À l'opposé, le site de Koro, le plus dégradé, n'a permis d'observer que des espèces très anthropophiles, dont $R$. rattus généralement commensal. Les

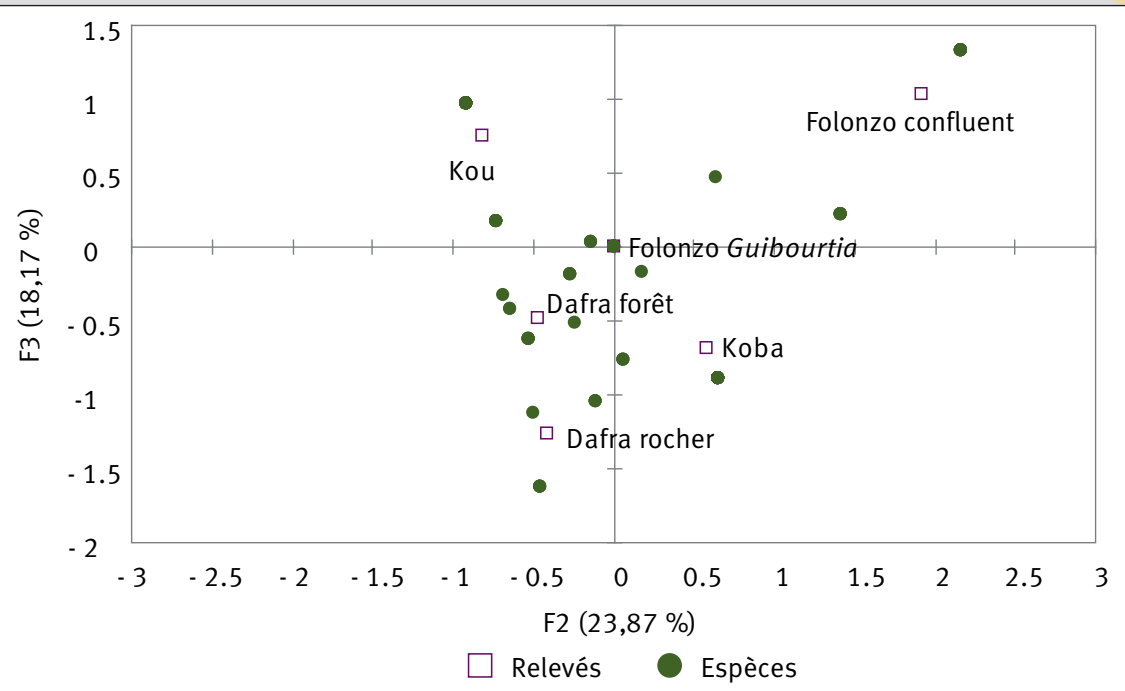

Figure 6.

Typologie de la végétation de six formations de forêt dense, axes 2 et 3. L'axe 3 classe les formations ripicoles en fonction du développement de la forêt : forêts-galeries (Kou et Folonzo confluent), galeries forestières plus étroites (Dafra forêt et Koba), galerie résiduelle sur rocher (Dafra rocher).

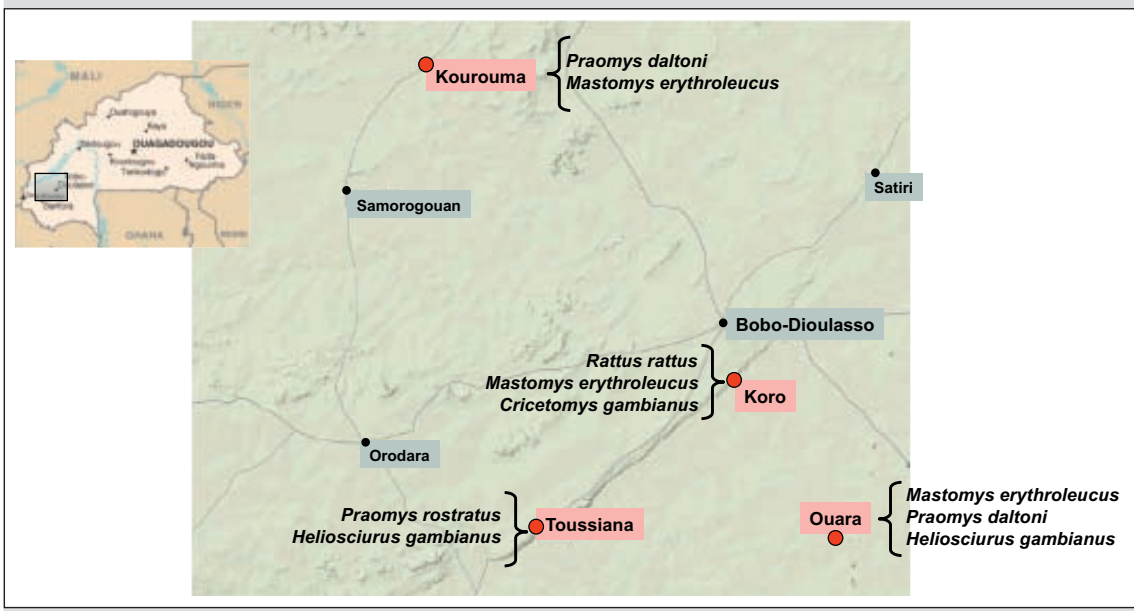

Figure 7.

Répartition des micromammifères dans les galeries

forestières plus ou moins dégradées de la falaise de Banfora

(Toussiana et Koro) et de ses environs (Ouara et Kourouma). communautés de petits mammifères des sites forestiers échantillonnés à Ouara, dominées par l'espèce généraliste Mastomys erythroleucus, semblent également avoir subi les conséquences des perturbations fortes observables dans ces habitats : réduction de la largeur des galeries forestières, transitions brusques avec des zones de savanes arbustives ou arborées très dégradées, importance des activités anthropiques (coupe de bois, cultures, pâturage). Toutes ces pressions semblent un peu moins fortes dans la zone de Kourouma, où se maintient apparemment une ripisylve relativement préservée, donc une mosaïque d'habitats plus harmonieuse, permettant la présence équilibrée de Praomys daltoni Thomas, espèce à tendance forestière, et de $M$. erythroleucus (Granjon et al., 2009). 
guerini Janson, Plaesiorrhinella cinctuta diaphana Voet, Charaxes tiridates tiridates et Charaxes brutus brutus, mais aussi des espèces non rencontrées plus au sud, comme Eudicella daphnis Buquet ou Euphaedra medon medon Linné. Cette forêt bien conservée pourrait alors servir de source pour une partie des espèces retrouvées sur la falaise, comme Chlorocala guerini, Chlorocala africana, Eudicella daphnis, Charaxes tiridates tiridates, Charaxes numenes numenes et Charaxes brutus brutus. Toutes les espèces provenant du bloc ivoirien n'y parviennent cependant pas puisque Charaxes northcotti Rotschild n'est trouvée qu'à Folonzo et sur la falaise, Charaxes lactetinctus lactetinctus Karsch à Folonzo uniquement et Stephanorhina guttata à Toussiana uniquement. Cette forêt présente également une grande espèce de cétoine, Dicronorhina kouensis Legrand, Juhel, Bouyer \& Camiade, qui n'est par ailleurs rencontrée que plus à l'est, dans des forêts-galeries du Togo et du Bénin (Legrand et al., 2006). Bebearia senegalensis Herrich Schäffer n'est connue quant à elle, que de forêts-galeries situées plus à l'ouest, en Guinée et au Sénégal.

Enfin, la falaise de Banfora présente une espèce qui se trouve ici à l'extrémité est de son aire; Coeliades aeschilus, par ailleurs rencontrée en Guinée et au Mali, et qu'on ne retrouve pas dans la forêt du Kou.

Beaucoup d'autres espèces forestières communes à ces relictes et à la forêt du Kou sont rencontrées également, constituant tout de même une part importante de la biodiversité entomologique du Burkina, ce qui renforce l'intérêt de conserver ces sites.

Les résultats confirment la nécessité de préserver ces ressources génétiques exceptionnelles, avant qu'il ne soit trop tard.

\section{COTE D'IVOIRE}

Figure 8.

L'origine supposée du peuplement entomologique (Cetoniinae, ${ }^{\star}$ Nymphalidae et ${ }^{\star \star}$ Hesperoideae) de la falaise de Banfora. Toutes les espèces présentées sont forestières, à l'exception de Charaxes northcotti, Charaxes lactetinctus lactetinctus et Coeliades aeschylus, qui sont des espèces rares de savane guinéenne

(sources : AlLard, 1991 ; De PALMA, 2009 ; LARSEN, 2006 ; LEGRAND et al., 2006 ; SAKAY, NAGAI, 1998).

N.B. : Les espèces botaniques citées sont conservées à l'herbier du Cirdes à BoboDioulasso. Les doubles sont déposés à l'herbier du Cirad (code Alf) à Montpellier (Campus international de Baillarguet, F34398 Montpellier Cedex 05, France).

\section{Les insectes}

La figure 8 cartographie la même démarche pour quelques espèces d'insectes typiques, afin de tenter d'expliquer l'origine éventuelle du peuplement de la falaise de Banfora. Une situation similaire à ce qui est observé pour les plantes s'observe chez les insectes.

La forêt classée du Kou à Dindéresso présente la plus grande richesse. La plupart des espèces semblent provenir du bloc forestier ivoirien, avec un relais possible par la forêt de Diéfoula pour certaines espèces, comme Chlorocala

\section{Remerciements :}

Les auteurs remercient l'Ambassade de France à Ouagadougou pour le financement du projet Fsp 2002-87 "Gestion durable des ressources sylvo-pastorales et production fourragère dans l'Ouest du Burkina-Faso » qui a permis de réaliser cette étude.

Les remerciements vont aussi à Jakob Fahr et Natalie Weber (Université d'Ulm, Allemagne) pour leur aide à l'étude des chauves-souris. 


\section{RÉFÉRENCES BIBLIOGRAPHIQUES}

ADJANOHOUN E., 1964. Végétation des savanes et des rochers découverts en Côte-d'Ivoire centrale. Paris, France, Orstom, coll. Mémoires, $\mathrm{n}^{\circ}$ 7, $178 \mathrm{p}$.

ALEXANDRE D. Y., 1978. Le rôle disséminateur des éléphants en forêt de Taï, Côte d'Ivoire. La Terre et la Vie, 32 (1) : 47-72. AUBRÉVILLE A., 1949. Climats, forêts et désertification de l'Afrique tropicale. Paris, France, Société d'éditions géographiques maritimes et coloniales, $352 \mathrm{p}$.

AUBRÉVILLE A., 1950. Flore forestière soudano-guinéenne. Paris, France, Société d'éditions géographiques maritimes et coloniales. Réédité en 1983 par le Ctft, Nogent-sur-Marne, France, $522 \mathrm{p}$.

AUBRÉVILLE A., 1958. À la recherche de la forêt en Côted'Ivoire. Bois et forêts des tropiques, 57 : 11-27.

AUBRÉVILLE A., 1959. La flore forestière de la Côte-d‘Ivoire. Nogent-sur-Marne, France, Centre technique forestier tropical, $2^{\mathrm{e}}$ édition, 3 vol., $1050 \mathrm{p}$.

BÉGUÉ L., 1937. Contribution à l'étude de la végétation forestière de la haute Côte d'Ivoire. Publication du Comité d'études historiques et scientifiques de l'Afrique de l'Ouest Française, Paris, série B, $1: 127 \mathrm{p}$.

BOUDET G., LEBRUN J.-P., 1986. Catalogue des plantes vasculaires du Mali. Maisons-Alfort, France, lemvt, Études et synthèses, $n^{\circ} 16,486 \mathrm{p}$.

BOUYER J., BALENGHIEN T., RAVEL S., VIAL L., SIDIBE I., THEVENON S., SOLANO P., DE MEEUS T., 2009. Population sizes and dispersal pattern of tsetse flies: rolling on the river? Molecular Ecology, 18 (13): 2787-2797.

CUISANCE D., POLITZAR H., MEROT P., TAMBOURA I., 1984. Les lâchers de mâles irradiés dans la campagne de lutte intégrée contre les glossines dans la zone pastorale de Sidéradougou, Burkina Faso. Revue d'Elevage et de Médecine vétérinaire des Pays tropicaux, 37 : 449-468.

GARDINER A. J., REID R. S., KIÉMA S., 2005. Impact of landuse on butterflies in south-western Burkina Faso. African Entomology, 13: 201-212.

GAUTUN J.-C., TRANIER M., SICARD B., 1985. Liste préliminaire des rongeurs du Burkina Faso. Mammalia, 49 : 537-542.

GRANJON L., ABDOULAYE D., AG ABOUMAHAMAD I., 2009. Rapport final « Micro-mammifères » du projet Fsp 2002-87 «Gestion durable des ressources sylvo-pastorales et production fourragère dans l'Ouest du Burkina Faso ». Ird, Umr 022, 8 p. GUINKO S., 1985. Contribution à l'étude de la végétation et de la flore du Burkina Faso. Les reliques boisées ou bois sacrés. Bois et forêts des tropiques, $208:$ 29-36.

GUINKO S., 2005. Flore illustrée de la forêt classée du Kou. Projet d'aménagement participatif des forêts classées de Dindéresso et du Kou, Ouagadougou, Burkina Faso, ministère de l'Environnement et du Cadre de Vie, 143 p.

HANSKI I., GAGGIOTII O. E., 2004. Ecology, genetics and evolution of metapopulations. San Diego, États-Unis, Elsevier Academic Press, 696 p.

HIERNAUX P., 1975. Étude phyto-écologique des savanes du pays baoulé méridional (Côte-d'Ivoire centrale). Thèse de doctorat, Université des sciences et techniques du Languedoc, Montpellier, France, 206 p.
HUTCHINSON J., DALZIEL M. D., 1954 à 1972. Flora of West tropical Africa. Second edition London, Millbank, Crown Agent for Oversea Governments and Administrations, vol. I part 1, 1954, 295 p.; vol. I part 2, 1958, 296-828; vol. II, 1963, 544 p.; vol. III part 1, 1968, 276 p.; vol. III part 2, 1972, 277-574.

JAEGER P., 1956. Contribution à l'étude des forêts reliques du Soudan occidental. Bulletin de l'Institut Français d'Afrique Noire, 18 A (4) : 993-1053.

KIÉMA S., 2007. Élevage extensif et conservation de la diversité biologique dans les aires protégées de l'Ouest burkinabé. Arrêt sur leur histoire, épreuves de la gestion actuelle, état et dynamique de la végétation. Thèse de doctorat, université d'Orléans, France, 658 p.

KIÉMA S., FOURNIER A., 2007. Utilisation de trois aires protégées par l'élevage extensif dans l'Ouest du Burkina Faso. In : Fournier A., Sinsin B., Mensah G. A. (éd.). Quelles aires protégées pour l'Afrique de l'Ouest ? Conservation de la biodiversité et développement. La biodiversité et les hommes. Paris, France, Ird, coll. Colloques et séminaires, 498-506.

LARSEN T. B., ADUSE-POKÚ K., SÁFIÁN S., 2009. The Butterflies of Boabeng-Fiema Monkey Sanctuary - Biodiversity and extinction in a forest fragment in Ghana. African Entomology, 17 (2): 131-146.

LEBRUN J.-P., TOUTAIN B., GASTON A., BOUDET G., 1991. Catalogue des plantes vasculaires du Burkina Faso. MaisonsAlfort, France, lemvt, Études et synthèses, $n^{\circ}$ 40, 341 p.

LEGRAND J.-P., JUHEL P., BOUYER J., CAMIADE D., 2006. Une nouvelle espèce de Dicronorhina Hope, du Burkina Faso, du Bénin et du Togo. Cahiers Magellanes hors-série $n^{\circ} 23,10 p$.

NASI R., SABATIER M., 1988. Projet inventaire des ressources ligneuses au Mali. Rapport de synthèse, première phase. Les formations végétales. Scet Agri/Ctft, $197 \mathrm{p}$.

NICOLAS V., BRYJA J., AKPATOU B., KONECNY A., LECOMPTE E., COLYN M., LALIS A., COULOUX A., DENYS C., GRANJON L., 2008. Comparative phylogeography of two sibling species of forest-dwelling rodent (Praomys rostratus and $P$. tullbergi) in West Africa: Different reactions to past forest fragmentation. Molecular Ecology, 17: 5118-5134.

PELTRE P., 1977. Le « V Baoulé » (Côte d’Ivoire centrale). Héritage géomorphologique et paléoclimatique dans le tracé du contact forêt-savane. Paris, France, Orstom, coll. Travaux et documents, $n^{\circ} 80,198 \mathrm{p}$.

SCHNELL R., 1971. Introduction à la phytogéographie des pays tropicaux. 2. Les milieux, les groupements végétaux. Paris, France, Gauthier-Villars, 951 p.

TEERWENBERG A. J. M., 1965. The Loganiaceae of Africa, Strychnos II. Acta Botanica Neerlandica, 14: 218-229.

VON LOTHAR S., 1982. Roloffia banforensis spec. nov. aus Obervolta. Die Aquarien und Terrarien Zeitschrift, 12: 448-454. 